\title{
Cryptanalysis of a Digital Signature Scheme on ID-Based Key-Sharing Infrastructures
}

\author{
Hongjun Wu, Feng Bao, and Robert H. Deng \\ Kent Ridge Digital Labs \\ 21 Heng Mui Keng Terrace, Singapore 119613 \\ \{hongjun, baofeng, deng\}@krdl . org.sg
}

\begin{abstract}
At ISW'99, Nishioka, Hanaoka and Imai proposed a digital signature scheme on ID-based key-sharing infrastructures. That signature scheme is claimed to be secure if the discrete logarithm problem is hard to solve. Two schemes (the ID-type and the random-type schemes) based on the linear scheme for the Key Predistribution Systems (KPS) and the discrete logarithm problem (DLP) were given.

In this paper we show that those two schemes fail to meet the nonrepudiation requirement: with negligible amount of computation, a signature could be forged. For the ID-type signature scheme, any verifier could forge a signature to raise repudiation between that verifier and the signer. The random type signature scheme has the same weakness. Furthermore, for the random-type signature scheme, once a signer issued a signature, anyone (not only the user in the scheme) could forge that signer's signature for a $\mathrm{n}$ arbitrary message.
\end{abstract}

\section{Introduction}

Digital signature plays an important role in authenticating digital documents. The commonly used digital signature schemes [511,13] are all belong to the public key cryptosystem (PKC). There is a kind of digital signature scheme that is based on the ID-Based cryptosystem 14 instead of on PKC. The first such digital signature scheme was proposed by Shamir [14. Some other digital signature schemes 310 12 are based on the Key Predistribution Systems (KPS). The KPS is a kind of ID-based cryptosystem and it solves the key distribution problem with simple calculation 1246 6/8 9 .

Nishioka, Hanaoka and Imai recently proposed a new signature scheme 12 . on the KPS infrastructure. This scheme is to satisfy the main signature requirements: authenticity, unforgeability, and non-repudiation [12]. Two examples were given and they are claimed to be secure if the discrete logarithm problem is hard to solve. As will be shown in this paper, those two examples are insecure: a signature could be easily forged with negligible amount of computation.

Nishioka, Hanaoka and Imai also claimed that if an ID-based key sharing system exists, an ID-based digital signature system could be easily implemented [12]. By breaking their proposed concrete signature schemes, it is shown that 
the implementation of secure ID-based digital signature system might not be as easy as claimed in 12 .

This paper is organised as follows. Section 2 introduces the KPS and the linear KPS scheme. Section 3 introduces the KPS based signature scheme. The attacks against the ID-type signature scheme and the random-type signature scheme are given in Section 4 and Section 5, respectively. Section 6 concludes this paper.

\section{KPS and the Linear KPS Scheme}

\section{$2.1 \quad$ KPS}

The KPS Key Predistribution System [9] consists of one centre and a number of users. The KPS centre keeps in secret a bi-symmetric "center-algorithm" $G(\cdot, \cdot)$. The centre computes each user's secret algorithm as $X_{i}(\cdot)=G\left(I D_{i}, \cdot\right)$, where $I D_{i}$ is the identifier of the user $U_{i}$ and is publicly authenticated. The algorithm $X_{i}$ is pre-distributed to the user $U_{i}$ secretly and confidentially. The user $U_{i}$ could establish a secret common key $k_{i j}$ with the user $U_{j}$ by computing $k_{i j}=X_{i}\left(I D_{j}\right)$ (the user $U_{j}$ computes it as $X_{j}\left(I D_{i}\right)$ ).

\subsection{The Linear KPS Scheme}

The linear KPS scheme is one of the basic schemes of the KPS. In this scheme the symmetric centre algorithm is represented as 2nd degree covariant tensor $G$ where each element $G_{i j}(i, j=0, \cdots, m-1)$ is in $G F(q)$. A public function $f$ transforms each ID into an $m$-dimension vector $x$ on $G F(q)$ as $x_{i}=$ $f\left(I D_{i}\right)$, where $x_{i}=\left(x_{i}^{0}, x_{i}^{1}, \cdots, x_{i}^{m-1}\right)^{T}$. The user $U_{i}$ 's secret algorithm is an $m$-dimension vector $X_{i}$ over $G F(q)$ and is generated as

$$
X_{i}=G \cdot x_{i}
$$

The common key $k_{i j}$ established between $U_{i}$ and $U_{j}$ is computed by $U_{i}$ as

$$
k_{i j}=X_{i}^{T} \cdot x_{j}
$$

or computed by $U_{j}$ as

$$
k_{i j}=X_{j}^{T} \cdot x_{i}
$$

As long as the number of users is less than $m$, this linear KPS scheme is secure since no user(s) could recover the centre algorithm $G$.

\section{The KPS-Based Signature Scheme}

In [12, two examples based on the linear scheme for the KPS and the discrete logarithm problem (DLP) are given. These two signature schemes are introduced in this section. In the rest of this paper, $H$ denotes an ideal hash function. 


\subsection{The ID-Type Scheme}

Choose two sufficiently large primes $p$ and $q$ such that $p=2 q+1$.

Signature Generation. The user $U_{i}$ signs a message $M$ as follows.

1. Compute $h=H\left(M \| I D_{i}\right) \bmod p$

2. The signature $S$ is an $m$-dimension vector on $G F(p)$ computed as $S=h^{X_{i}}$, i.e., $S_{\ell}=h^{X_{i}^{\ell}} \bmod p$ for $\ell=0,1, \cdots, m-1$.

Verification. The user $U_{j}$ verifies the signature as follows:

1. Compute $V_{1}=\prod_{\ell=0}^{m-1}\left(S_{\ell}\right)^{x_{j}^{\ell}} \bmod p$

2. Compute $V_{2}=h^{k_{i j}} \bmod p$ where $k_{i j}$ is the common key shared by $U_{i}$ and $U_{j}$.

3. If $V_{1}=V_{2}$ or $V_{1}=V_{2} \cdot h^{q} \bmod p$, the signature is accepted; otherwise, it is rejected.

Remarks. In the signature verification process,

$$
\begin{aligned}
V_{1} & =\prod_{\ell=0}^{m-1}\left(S_{\ell}\right)^{x_{j}^{\ell}} \bmod p \\
& =h^{\sum_{\ell=0}^{m-1} X_{i}^{\ell} \cdot x_{j}^{\ell} \bmod p-1} \bmod p \\
& = \begin{cases}h^{k_{i j}} \bmod p & \text { if } \widetilde{k}_{i j}<q \\
h^{k_{i j}} \cdot h^{q} \bmod p \text { otherwise }\end{cases}
\end{aligned}
$$

where $\widetilde{k}_{i j}=\sum_{\ell=0}^{m-1} X_{i}^{\ell} \cdot x_{j}^{\ell} \bmod p-1$. Thus for a signature generated by $U_{i}, V_{1}=V_{2}$ or $V_{1}=V_{2} \cdot h^{q} \bmod p$.

\subsection{The Random-Type Scheme}

Choose two sufficiently large primes $p$ and $q$ satisfying $q \mid p-1$. Pick up $g$ with order $q$ on the multiplicative group $\mathbf{Z}_{p}^{*}$.

Signature Generation. The user $U_{i}$ signs the message $M$ as follows:

1. Generate $m$ random numbers $z_{\ell} \in \mathbf{Z}_{q}(\ell=0,1, \cdots, m-1)$.

2. Let $z=\sum_{\ell=0}^{m-1} z_{\ell} \bmod q$, and $r_{\ell}=g^{z_{\ell}} \bmod p$.

3. Compute $s_{\ell}=\left(H(M) \cdot z_{\ell}+X_{i}^{\ell}\right) \cdot z^{-1} \bmod q$.

4. The signature of the message $M$ is given as $\left(r_{\ell}, s_{\ell}\right)(\ell=0,1, \cdots, m-1)$. 
Verification. The user $U_{j}$ verifies the signature as follows:

1. Let $s=\sum_{\ell=0}^{m-1} s_{\ell} \cdot x_{j}^{\ell} \bmod q$, and $r=\prod_{\ell=0}^{m-1} r_{\ell}^{x_{j}^{\ell}} \bmod p$.

2. Let $k_{i j}=\sum_{\ell=0}^{m-1} X_{j}^{\ell} \cdot x_{i}^{\ell} \bmod q$.

3. Compute $V_{1}=r_{m-1}^{H(M) s^{-1}} \cdot g^{k_{i j} \cdot s^{-1}} \bmod p$.

4. Compute $V_{2}=\prod_{\ell=0}^{m-1} r_{\ell} \bmod p$.

5. The user $U_{j}$ accepts the signature only if $V_{1}=V_{2}$.

Remarks. It is not difficult to verify that for a signature generated by $U_{i}$, $V_{1}=V_{2}$.

\section{Cryptanalysis of the ID-type Signature Scheme}

In this section, we show that it is easy for a verifier to forge a signature. This signature could pass this verifier's verification process while it could not pass the verification processes of the other verifiers. However it is sufficient to cause repudiation between the signer and the verifier since the signer, on the other hand, can also forge such a signature to cheat that verifier.

Suppose now the signer is $U_{i}$ and the verifier is $U_{j}$. With only the knowledge of $k_{i j}$, either the user $U_{i}$ (signer) or $U_{j}$ (verifier) could forge the signature as follows:

1. Compute $h=H\left(M \| I D_{i}\right)$ and $V_{2}=h^{k_{i j}} \bmod p$.

2. Solve for $a_{\ell}(\ell=0,1, \cdots, m-1)$ from the following equation:

$$
V_{2}=\prod_{\ell=0}^{m-1} h^{a_{\ell} \cdot x_{j}^{\ell}} \bmod p, \text { or } V_{2} \cdot h^{q}=\prod_{\ell=0}^{m-1} h^{a_{\ell} \cdot x_{j}^{\ell}} \bmod p
$$

3. Let $S_{\ell}^{\prime}=h^{a_{\ell}} \bmod p . S_{\ell}^{\prime}(\ell=0,1, \cdots, m-1)$ are the forged signature.

In step 2, one of the equation is always solvable. And the amount of computation used in this attack is negligible. Thus either the verifier or signer could forge the signature easily.

The attack above deals with only one verifier. In the following, we consider the case in which all the verifiers are involved. All the verifiers may collude to forge a signature that could pass every verifier's verification. And the signer can also forge such a signature to cheat all the verifiers. Suppose there are $n(n<m)$ users in the KPS scheme. The user $U_{0}$ is the signer and the users $U_{1}$ to $U_{n-1}$ are the verifiers. In the KPS scheme, the signer has the knowledge of the common keys $k_{0 j}(j=1,2, \cdots, n-1)$. The colluded verifiers could also obtain the information of such common keys. The following attack shows how such a signature could be forged with the knowledge of the common keys $k_{0 j}(j=1,2, \cdots, n-1)$. 
1. Compute $h=H\left(M \| I D_{0}\right)$. Let $V_{2 j}=h^{k_{0 j}} \bmod p$ for $j=1,2, \cdots, n-1$.

2. Solve for $a_{\ell}(\ell=0,1, \cdots, m-1)$ from the following equations:

$$
V_{2 j}=\prod_{\ell=0}^{m-1} h^{a_{\ell} \cdot x_{j}^{\ell}} \bmod p,\left(\text { or } V_{2 j} \cdot h^{q}=\prod_{\ell=0}^{m-1} h^{a_{\ell} \cdot x_{j}^{\ell}} \bmod p\right)(j=1,2, \cdots, n-1)
$$

3. Let $S_{\ell}^{\prime}=h^{a_{\ell}} \bmod p . S_{\ell}^{\prime}(\ell=0,1, \cdots, m-1)$ are the forged signature.

\section{Cryptanalysis of the Random-Type Signature Scheme}

The random-type signature scheme is more vulnerable than the ID-type scheme. In this scheme, every verifier could forge the signature. Furthermore, anyone (not only the users in the scheme) could forge a signer's signatures after that signer released a signature. In Subsection 5.1, we show how a verifier could forge a signature. In Subsection 5.2, we show how a signer's signature could be forged after one signature is issued.

\subsection{A Verifier Could Forge the Signature}

With the knowledge of the common key $k_{i j}$, either the user $U_{i}$ (signer) or $U_{j}$ (verifier) could forge the signature as follows:

1. Choose $m$ random numbers $b_{i}(i=0,1, \cdots, m-1)$ from $G F(q)$. Let $r_{i}=g^{b_{i}}$.

2. Let $r=\prod_{\ell=0}^{m-1} r_{\ell}^{x_{j}^{\ell}} \bmod p, v=\prod_{\ell=0}^{m-1} r_{\ell} \bmod p$.

3. Solve the following equation for $s$ :

$$
v=r^{H(M) \cdot s^{-1}} \cdot g^{k_{i j} \cdot s^{-1}} \bmod p
$$

4. Choose $m$ numbers $s_{\ell}(\ell=0,1, \cdots, m-1)$ to satisfy the following linear equation:

$$
s=\sum_{\ell=0}^{m-1} s_{\ell} \cdot x_{j}^{\ell} \bmod q
$$

5. $\left(r_{\ell}, s_{\ell}\right)(\ell=0,1, \cdots, m-1)$ are the forged signature.

Remarks In step 3, the equation could be solved without dealing with the discrete log problem. The reason is that we only need to deal with the exponent since all the bases are the same $(g)$. The amount of computation used in the attack is negligible. 


\subsection{Anyone Could Forge the Signature}

After a signer issued a signature, his signatures could be forged by anyone (not only the user in the KPS scheme). Suppose that a user $U_{i}$ has issued a signature $S$ of message $M$. We show that anyone could forge the signature of an arbitrary message $M^{\prime}$ and to convince a verifier $U_{j}$ that the signature is given by $U_{i}$. The attack is given as follows:

1. Recover $g^{k_{i j}}$ from the signature: from the verification process of signature, it is easy to compute the value of $g^{k_{i j}}$ from the signature and the publicly known information.

2. Let $g^{\prime}=g^{k_{i j}}$.

3. Choose $m$ random numbers $b_{i}(i=0,1, \cdots, m-1)$ from $G F(q)$. Let $r_{i}^{\prime}=g^{\prime b_{i}}$.

4. Let $r^{\prime}=\prod_{\ell=0}^{m-1} r^{\prime} x_{j}^{\ell} \bmod p, v^{\prime}=\prod_{\ell=0}^{m-1} r_{\ell}^{\prime} \bmod p$.

5. Solve the following equation for $s^{\prime}$ :

$$
v^{\prime}=r^{\prime H\left(M^{\prime}\right) \cdot s^{\prime-1}} \cdot g^{\prime s^{\prime-1}} \bmod p
$$

6. Choose $m$ numbers $s_{\ell}^{\prime}(\ell=0,1, \cdots, m-1)$ to satisfy the following linear equation:

$$
s^{\prime}=\sum_{\ell=0}^{m-1} s_{\ell}^{\prime} \cdot x_{j}^{\ell} \bmod q
$$

7. $\left(r_{\ell}^{\prime}, s_{\ell}^{\prime}\right)(\ell=0,1, \cdots, m-1)$ are the forged signature.

Remarks In step 5, the equation could be solved easily since the bases are all the same $\left(g^{\prime}\right)$, and only the exponent need to be considered.

Conclusion $=====================$

\section{Conclusions}

In this paper, we showed that two recently proposed KPS-based signature schemes are not secure. The design of secure signature scheme based on KPS is still an open problem.

\section{References}

1. R. Blom, "Non-public Key Distribution", in Advances in Cryptology-Crypto'82, Plenum Press (1983), pp. 231-236.

2. C. Blundo, A. De Santis, A. Herzberg, S. Kutten, U. Vaccaro and M. Yung, "Perfectly Secure Key Distribution for Dynamic Conferences", in Advances in Cryptology-Crypto'92, LNCS 740, Springer-Verlag, pp.471-486, 1993.

3. Y. Desmedt and J. J. Quisquater, "Public-Key Systems Based on the Difficulty of Tampering (Is There a Difference Between DES and RSA?)", in Advances in Cryptology Crypto'86, LNCS 263, Springer-Verlag, pp. 111-117, 1986. 
4. Y. Desmedt and V. Viswanathan, "Unconditionally Secure Dynamic Conference Key Distribution”, IEEE, ISIT'98, 1998.

5. T. ElGamal. "A Public Key Cryptosystem and a Signature Scheme based on Discrete Logarithms". IEEE Transactions on Information Theory, 31 (1985), 469-472.

6. A. Fiat and M. Naor, "Broadcast Encryption", in Advances in CryptologyCrypto'93, LNCS 773, Springer-Verlag, pp. 480-491, 1994.

7. L. Gong and D. J. Wheeler, "A Matrix Key-Distribution Scheme", Journal of Cryptology, vol. 2, pp. 51-59, Springer-Verlag (1993).

8. W. A. Jackson, K. M. Martin, and C. M. O'keefe, "Multisecret Threshold Schemes", in Advances in Cryptology-Crypto'93, LNCS773, Springer-Verlag, pp. 126-135, 1994.

9. T. Matsumoto and H. Imai, "On the Key Predistribution System: A Practical Solution to the Key Distribution Problem", in Advances in Cryptology-Crypto'87, LNCS 293, Springer-Verlag, pp.185-193, 1987.

10. T. Matsumoto and H. Imai, "Applying the Key Predistribution Systems to Electronic Mails and Signatures", in Proc. of SITA'87, pp. 101-106, 1987.

11. V. Miller, "Uses of Elliptic Curves in Cryptography", in Advances in CryptologyCrypto'85, LNCS 218, Springer-Verlag, pp. 417-426, 1986.

12. T. Nishioka, G. Hanaoka, and H. Imai, "A New Digital Signature Scheme on ID-Based Key-sharing Infrastructures", in Information Security-Proc. of ISW'99, LNCS 1729, Springer-Verlag, pp. 259-270, 1999.

13. R. L. Rivest, A, Shamir, and L. Adleman, "A method for Obtaining Digital Signatures and Public-Key Cryptosystems", Commun. ACM, vol. 21, no. 2, pp. 158-164, Feb. 1978.

14. A. Shamir, "Identity-Based Cryptosystems and Signature Schemes", in Advances in Cryptology-Crypto'84, LNCS 196, Springer-Verlag, pp. 47-53, 1985. 